\section{IN THE NEWS}

Smallpox vaccine safety The United States government stopped routine smallpox vaccination in 1970 , but the possibility of a bioterrorist attack led to resumption of vaccination for health-care workers this year. However, the Advisory Committee on Immunization Practices has now recommended that vaccination should not be extended owing to cardiac complications in some military personnel, who were vaccinated before the war in Iraq (Washington Post). Controversy over safety of the vaccine has been increased by proposals for its use to combat the recent outbreak of related monkeypox. The Centers for Disease Control and Prevention (CDC) is recommending vaccination for all those thought to have been exposed to monkeypox. As the smallpox vaccine consists of live vaccinia virus, it can have serious side effects. Paul Fine of the London School of Hygiene \& Tropical Medicine says that important decisions must be made about the risks and benefits of vaccination (New Scientist), and CDC guidelines state that patients must give informed consent. Ron Atlas - President of the American Society for Microbiology went one step further in a recent talk by suggesting that vaccinia and monkeypox viruses could recombine, thereby creating a more deadly strain.

Safety issues are clearly of concern to those being offered vaccination. In Madison, Wisconsin, of about 90 people who were told they were eligible for vaccination after exposure to monkeypox none showed up at the clinic. And and Ohio and Illinois have decided not to offer vaccination (New York Times). At the moment, the number of people involved is small, but Fine suggests that if the virus becomes endemic in wild rodent populations, the number of human cases will rise and the controversy over vaccination will become more significant (The Guardian).

Kirsty Minton

\title{
Hide and seek
}

My favourite hiding place as a kid involved wrapping myself in the lounge curtains but, much to my frustration, it was always obvious to my sister where I was. To evade detection, some bacteria use a similar strategy of veiling themselves inside cells. However, just like big sis, the innate immune system is still able to detect these hideaways.

Toll-like receptors (TLRs) are known to be crucial for the detection of extracellular and phagocytosed bacteria, but how do cells sense invasive bacteria? NOD (nucleotide-binding oligomerization domain) proteins have been proposed as a new class of pattern-recognition receptor for cytoplasmic bacterial products, signalling through nuclear factor- $\mathrm{\kappa B}$ (NF- $\mathrm{\kappa B})$. Two studies, published in Science and Nature Immunology, have now looked in detail at the role of Nod 1 in the detection of intracellular bacteria.
It had previously been shown that commercial preparations of lipopolysaccharide (LPS) can activate Nod1. However, in these two studies, highly purified LPS was unable to stimulate this pathway, indicating that a contaminant might be responsible for Nod1 activation. Synthetic lipopeptides failed to stimulate Nod1, and carbohydrates and conventional polypeptides were shown not to be required for Nod 1 stimulation, so the investigation was focused on peptidoglycans (another component of bacterial cell walls). Analysis of digestion products of peptidoglycans refined the Nod 1 stimulus to a diaminopimelate (DAP)-containing $\mathrm{N}$-acetylglucosamine- $\mathrm{N}$-acetylmuramic acid motif (which is found in most Gram-negative bacteria and a small subset of Gram-positive bacteria). Chamaillard et al. stressed the importance of the DAP component

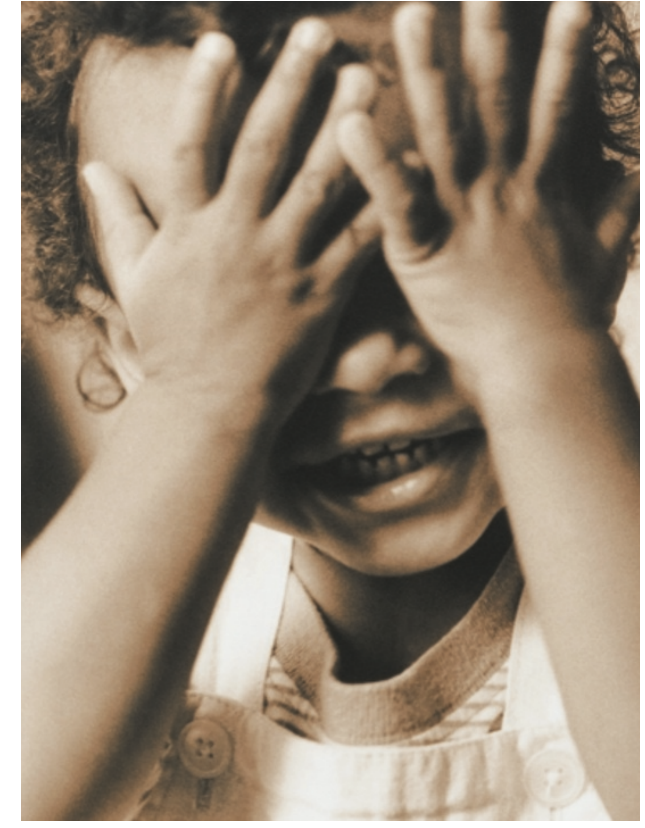

by showing that whereas synthetic DAP-containing peptides could stimulate Nod1, synthetic DAP-free counterparts could not.

The first cells to be encountered and entered by a large number of intracellular bacteria are epithelial cells, so Girardin et al. looked at the role of Nod 1 in these cells. They used a digitonin-based permeabilization technique to mediate entry of bacterial extracts into an epithelial cell line. Only extracts containing the DAP motif (mainly from Gram-negative

\section{MACROPHAGES}

\section{Orphan receptor finds a home}

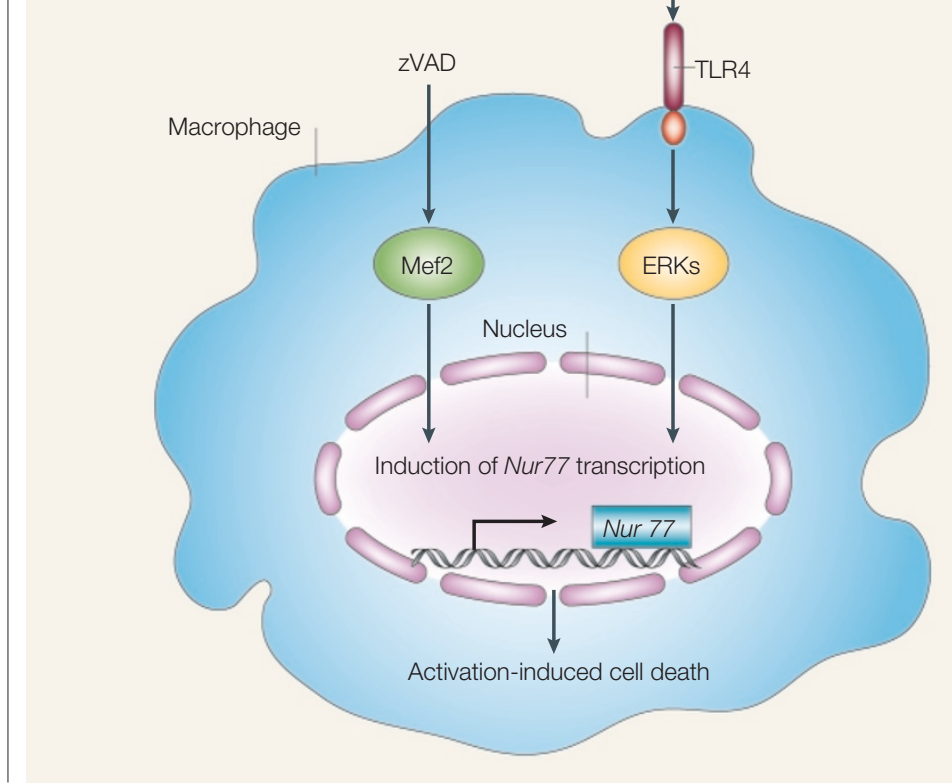

The molecular mechanisms underlying apoptosis or activationinduced cell death (AICD) of activated $T$ and $B$ cells are well understood. However, AICD also occurs in macrophages but, until now, the signalling pathways that are involved have remained unknown. Kim et al. report in The Journal of Experimental Medicine a crucial role for the orphan nuclear receptor NUR77 in macrophage cell death.

Activated macrophages are crucial for host defence against microbial pathogens, but their potential to cause tissue damage and inflammation means that they need to be tightly controlled; cell death is thought to be important in this regard.

Although caspases (cysteine proteinases) are required for the initiation and effector stages of some death pathways, AICD can also occur through caspaseindependent pathways. The authors of this study have 


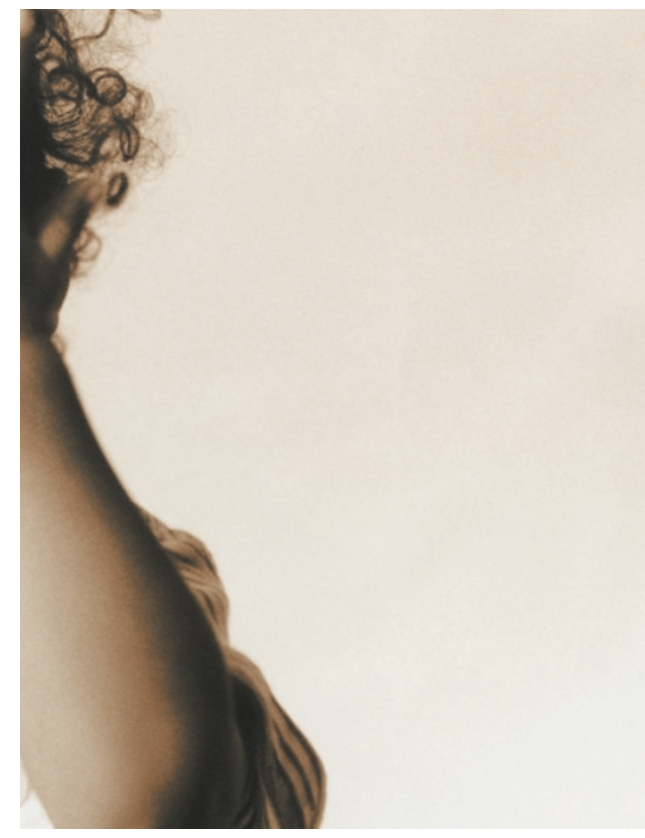

bacteria) could stimulate the Nod1NF- $\kappa \mathrm{B}$ pathway. Using the same method, intracellular presentation of Gram-negative bacterial supernatants to isolated intestinal epithelial cells from Nod1-deficient mice did not activate the NF- $\kappa B$ pathway, which indicates that Nod1 has a crucial role in the response of epithelial cells to this subset of bacteria. Similarly, Chamaillard et al. showed that Nodl is required for the macrophage response to intracellular bacteria, by looking at the ability of DAP-containing peptides to stimulate cytokine secretion by macrophages. Macrophages from Nod1-deficient mice failed to produce interleukin- 6 and tumournecrosis factor in response to these peptides.

So, it seems that there is no such thing as a safe hiding place for these bacteria. They can hide from TLRmediated detection by invading the cell cytoplasm, but are still spotted by the innate immune system through Nod1. Both groups speculate that in contrast to Nod2, which can recognize a broad range of bacteria, Nod1 is a subset-specific bacterial sensor (recognizing mainly Gram-negative bacteria). The functional relevance of this has yet to be determined.

Kirsty Minton

\section{(2) References and links} ORIGINAL RESEARCH PAPERS Girardin, S. E. et al. Nod1 detects a unique muropeptide from Gram-negative bacterial peptidoglycan. Science 300, 1584-1587 (2003) | Chamaillard, M. et al. An essential role for NOD1 in host recognition of bacterial peptidoglycan containing diaminopimelic acid. Nature Immunol. 8 June 2003 (DO) 10.1038/ni945)

FURTHER READING Inohara, N. \& Nuñez, G. NODS: intracellular proteins involved in inflammation and apoptosis. Nature Rev. Immunol. 3, 371-382 (2003) previously shown that costimulation of macrophages with lipopolysaccharide (LPS) - a virulence factor of Gram-negative bacteria - and the pan-caspase inhibitor zVAD resulted in caspaseindependent death of macrophages in vitro. Here, they confirmed these observations in vivo in a mouse sepsis model. Apoptosis of activated peritoneal macrophages was increased following treatment with zVAD. Signalling through Toll-like receptors TLR2 and TLR4 was shown to be required for macrophage death in this system.

Next, as zVAD-promoted macrophage death was not mediated by the classic caspase pathway for cell death, Kim et al. asked whether any death genes were induced during this process. Treatment of a mouse macrophage cell line with LPS and zVAD resulted in increased expression of Nur77 before cell death, indicating that this death receptor could trigger cell death in this setting.
This was confirmed by experiments with a putative dominant-active Nur77 mutant protein (which caused increased macrophage death) and with Nur77-deficient macrophages (which were less susceptible to cell death).

Detailed signalling analysis led the authors to propose that a dual signalling pathway leads to the induction of Nur77 expression by macrophages that are destined to undergo AICD. Activation of the extracellular signal-regulated kinase (ERK) pathway, which is downstream of TLR signalling, and activity of the myocyte-specific enhancer binding factor 2 (Mef2), which is upregulated by zVAD, were both required for the induction of Nur77 transcription and for Nur77mediated caspase-independent macrophage cell death.

Jenny Buckland

(0) References and links ORIGINAL RESEARCH PAPER Kim, S. O. et al. Orphan nuclear receptor Nur77 is involved in caspase-independent macrophage cell death. J. Exp. Med. 197, 1441-1452 (2003)

\section{IN BRIEF}

\section{LYMPHOCYTE ACTIVATION}

\section{Caspase activity is required for stimulated \\ B lymphocytes to enter the cell cycle.}

Olson, N. R. et al. J. Immunol. 170, 6065-6072 (2003)

Caspases are best known for their role in apoptosis, but they have also been implicated in the regulation of cell proliferation. In particular, caspase-8 - the factor that is at the top of the proteolytic caspase cascade - has a role in T-cell proliferation, but the specific caspases that are involved downstream are unknown. This new study shows that caspase- 8 and caspase- 6 are essential for human B-cell proliferation. Inhibition of caspase-6 blocked the upregulation of expression of the cell-cycle regulators D-type cyclins and cyclin-dependent kinase 4 by activated $\mathrm{B}$ cells, indicating a possible mechanism by which caspase- 6 could regulate cell-cycle entry. Notably, other aspects of B-cell activation, such as cytokine secretion, were unaffected, indicating a specific role for caspase- 6 in proliferation.

\section{LYMPHOCYTE ACTIVATION}

The B cell-specific major raft protein, Raftlin, is necessary for the integrity of lipid raft and BCR signal transduction.

\section{Saeki, K. et al. EMBO J. 22, 3015-3026 (2003)}

Membrane lipid microdomains known as rafts, which are rich in signalling molecules, have been proposed to have a crucial role in lymphocyte signalling. This study describes a new component of rafts in B cells, which has no homology to any other known protein and has been named Raftlin (for raft-linking protein). This molecule seems to localize in rafts by means of an acylated motif and is constitutively associated with the B-cell receptor (BCR), although it does not bind the BCR directly. In the absence of Raftlin, BCR signalling was impaired and levels of other key raft components, such as the tyrosine kinase LYN and ganglioside GM1, were markedly reduced, indicating that Raftlin is important for raft integrity .

\section{MUCOSAL IMMUNOLOGY}

\section{Mast cells disrupt epithelial barrier function during} enteric nematode infection.

McDermott, J. R. et al. Proc. Natl Acad. Sci. USA 100, 7761-7766 (2003)

Expulsion of nematode parasites is associated with elevated epithelial permeability. Mast cells are also important for parasite expulsion, but the mechanism is not well understood. In this study, McDermott and colleagues show that mast cells are responsible for inducing the increased intestinal permeability, which seems to depend on degradation of the tight-junction protein occludin. The authors used c-Kitspecific antibodies to block mast-cell accumulation, which prevented parasite expulsion and correlated with less permeability than in control animals. Mice overexpressing interleukin-9, which have higher numbers of mast cells than normal mice, showed enhanced intestinal permeability and could get rid of the parasites faster. 